\title{
Verhaltenstherapie
}

\section{Band 18, Heft 4, Dezember 2008}

Editorial

212450 Millionen Euro Sondermittel für Verhaltenstherapie - Ist die britische Initiative für psychisch Kranke ein Modell für Deutschland? Rief, W. (Marburg)

214 Dank an die Gutachter

Originalarbeiten

215 Bedeutung von Rumination und Ablenkung für den Therapieerfolg depressiver Patienten Teismann, T.; Willutzki, U.; Michalak, J.; Schulte, D. (Bochum)

223 Evaluation einer kognitiv-verhaltenstherapeutischen Intervention zur Reduktion von Prokrastination Höcker, A.; Engberding, M.; Beißner, J.; Rist, F. (Münster)

230 Stationsatmosphäre und Behandlungserfolg nach Eröffnung einer Spezialstation für Patienten mit Persönlichkeitsstörungen und akuten Krisen Uhlmann, C.; Steinert, T. (Ravensburg)

237 Reliabilität des Diagnostischen Interviews bei Psychischen Störungen (DIPS für DSM-IV-TR) unter klinischen Routinebedingungen Suppiger, A.; In-Albon, T.; Herren, C.; Bader, K.; Schneider, S.; Margraf, J. (Basel)

Originalarbeit - Kinder und Jugendliche

247 MIPAS-Family - Entwicklung und Evaluation eines verhaltensmedizinischen Programms zur Behandlung kindlicher Kopfschmerzen

Gerber, W.-D. (Kiel); Petermann, F. (Bremen); Gerber-von Müller, G.; Niederberger, U.; Rentmeister, B.; Siniatchkin, M.; Stephani, U. (Kiel)

Interview

257 Trudie Chalder: «Die Namen, die wir Krankheiten geben, sind wichtig». Ein Interview über chronische Erschöpfung Rief, W. (Marburg)
Vol. 18, Issue 4, December 2008

Editorial

212 A Special Grant of $\mathbf{4 5 0}$ Million Euro for Behavior Therapy - Could the British Initiative Be a Model for Germany? Rief, W. (Marburg)

214 Acknowlegement to the Reviewers

Original Articles

215 Relevance of Rumination and Distraction to Therapy Outcome in Depressed Patients

Teismann, T.; Willutzki, U.; Michalak, J.; Schulte, D. (Bochum)

223 Evaluation of a Cognitive-Behavioural Intervention for Procrastination

Höcker, A.; Engberding, M.; Beißner, J.; Rist, F. (Münster)

230 Ward Atmosphere and Treatment Success after Implementing a Specialised Hospital Ward for Patients with Personality Disorders and Acute Crises Uhlmann, C.; Steinert, T. (Ravensburg)

237 Reliability of the Structured Diagnostic Interview for Mental Disorders (DIPS for DSM-IV-TR) in Clinical Routine

Suppiger, A.; In-Albon, T.; Herren, C.; Bader, K.; Schneider, S.; Margraf, J. (Basel)

Original Article - Children and Adolescents

247 MIPAS-Family - Development and Evaluation of a Behavioural Medicine Programme for the Treatment of Chronic Paediatric Headaches

Gerber, W.-D. (Kiel); Petermann, F. (Bremen); Gerber-von Müller, G.; Niederberger, U.; Rentmeister, B.; Siniatchkin, M.; Stephani, U. (Kiel)

Interview

257 Trudie Chalder: 'Labels for Disorders are Highly Relevant'. An Interview about Chronic Fatigue Rief, W. (Marburg)

\section{KARGER}

Fax +497614520714

Information@Karger.de

www.karger.com 


\section{Verhaltenstherapie}

\section{Band 18, Heft 4, Dezember 2008}

261 Buchbesprechungen

265 Fort- und Weiterbildung

269 Mitteilungen der Verbände

273 Tagungen und Kongresse

275 Autorenverzeichnis Band 18, 2008

276 Sachwortverzeichnis Band 18, 2008

III Inhalt Band 18, 2008 (nach Seite 278)

II Impressum

U3 Hinweise für Autoren (3. Umschlagseite)

U2 Erläuterungen zum Titelbild (2. Umschlagseite) Leibl, C.; Austermann, T.; Antoni, S.; Naab, S. (Prien am Chiemsee)

Einen Ausblick auf den Inhalt der kommenden Hefte finden Sie auf Seite 278.

\section{Vol. 18, Issue 4, December 2008}

261 Book Reviews

265 Education

269 Information by Behavior Therapy Associations

273 Meetings and Conferences

275 Author Index Vol. 18, 2008

277 Subject Index Vol. 18, 2008

V Contents Vol. 18, 2008 (following page 278)

II Imprint

C3 Guidelines for Authors (Inside back cover)

C2 Explanations on the Title (Inside front cover) Leibl, C.; Austermann, T.; Antoni, S.; Naab, S. (Prien am Chiemsee)
Forthcoming papers are listed on page 278.

\section{KARGER}

Fax +497614520714

Information@Karger.de www.karger.com 